\title{
Current limiting characteristics of inductive type SCFCL with ZnO device and resistor in parallel
}

\section{AUTHOR(S):}

Shirai, Y; Morimoto, T; Furushiba, K; Shiotsu, M; Fushiki, K; Baba, J; Nitta, T

\section{CITATION:}

Shirai, Y...[et al]. Current limiting characteristics of inductive type SCFCL with ZnO device and resistor in parallel. IEEE TRANSACTIONS ON APPLIED SUPERCONDUCTIVITY 2006, 16(2): 654-657

\section{ISSUE DATE:}

2006-06

URL:

http://hdl.handle.net/2433/50268

\section{RIGHT:}

(c)2006 IEEE. Personal use of this material is permitted. However, permission to reprint/republish this material for advertising or promotional purposes or for creating new collective works for resale or redistribution to servers or lists, or to reuse any copyrighted component of this work in other works must be obtained from the IEEE. 


\section{Current Limiting Characteristics of Inductive Type SCFCL With ZnO Device and Resistor in Parallel}

Yasuyuki Shirai, Member, IEEE, Takayuki Morimoto, Kunimitsu Furushiba, Masahiro Shiotsu, Keisuke Fushiki, Junpei Baba, and Tanzo Nitta, Member, IEEE

\begin{abstract}
A new FCL system which consists of a L-type SCFCL in parallel with a resistor and a $\mathrm{ZnO}$ device is proposed. The proposed system limits the fault current inductively and also consumes excessive energy like R-type ones. A model system was made and studied experimentally. The $\mathrm{ZnO}$ device is off-state in stand-by mode. When a fault occurs, the SCFCL turns into current limiting mode and has high impedance. The voltage across the SCFCL exceeds a threshold voltage of the $\mathrm{ZnO}$ device. During one cycle of the voltage, the $\mathrm{ZnO}$ device is on-state around the peak of the voltage, and the current flows through both the SCFCL and the resistor. A certain amount of excessive energy is dissipated in the resistor. The $\mathrm{ZnO}$ device is off-state around the peak of the current which is suppressed only by the L-type SCFCL. Finally the proposed FCL system switches L-type and L+R-type in a cycle. Relations among the threshold voltage, the current limiting impedance and the resistance of the resistor were discussed. It was confirmed the proposed FCL system represents desirable current limiting characteristics.
\end{abstract}

Index Terms-Current limiting impedance, power system characteristics, power system stability, ZnO device.

\section{INTRODUCTION}

$\mathbf{S}$ UPERCONDUCTING Fault Current Limiters (SCFCLs) of various types have been proposed and tested [1]-[3]. It is important to study the power system characteristics of the SCFCLs. The authors designed and made a 3-phase SCFCL of transformer type with adjustable trigger current level for power system characteristics tests in a laboratory scale [4]. This model SCFCL is an inductive current limiting type. Trigger current level of each phase can be trimmed to be balanced. Excellent recovery characteristics were confirmed [5], [6]. Some demonstration tests on power system characteristics were carried out by use of a power system simulator and the model SCFCL [7], [8].

SCFCLs can be classified into two types by their current limiting impedance, that is, inductive-type (L-type) and resistivetype (R-type) FCL. Both types have advantages and disadvantages each other. The L-type FCL has good features in current limiting and recovery characteristics. The R-type one can consume the excessive energy of generators at a fault and it can improve power system stability.

Manuscript received September 20, 2005. This work was supported in part by the 21st Century COE program (No. 14219201), the MEXT Japan.

Y. Shirai, T. Morimoto, K. Furushiba, and M. Shiotsu are with the Department of Energy Science and Technology, Kyoto University, Yoshida-Honmachi, Sakyo-ku, Kyoto 606-8501, Japan (e-mail: shirai@energy.kyoto-u.ac.jp).

K. Fushiki, J. Baba, and T. Nitta are with the Department of Electrical Engineering, the University of Tokyo, 7-3-1, Hongo, Bunkyo-ku, Tokyo 113-8656, Japan (e-mail: baba@asc.t.u-tokyo.ac.jp; nitta@asc.t.u-tokyo.ac.jp).

Digital Object Identifier 10.1109/TASC.2006.870524
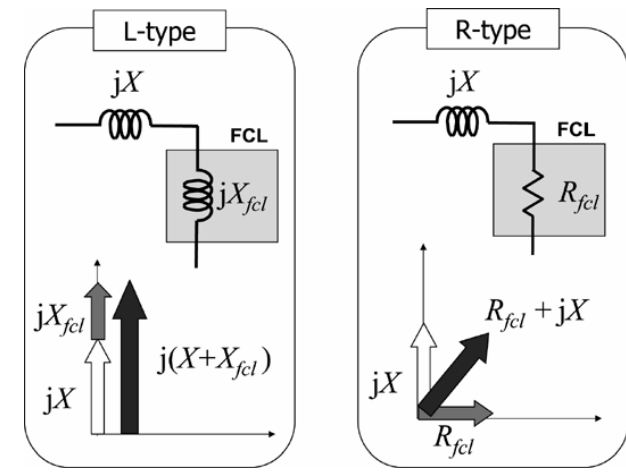

Fig. 1. Impedance of inductive and resistive FCL. Current limiting impedance $\left(R_{f c l}\right)$ of R-type FCL should be larger than that $\left(X_{f c l}\right)$ of L-type one for the same current limiting performance, since the impedance of a power system is normally inductive $(X)$.

In this paper, a new FCL system which consists of L-type SCFCL with a resistor and $\mathrm{ZnO}$ device in parallel is proposed. Current limiting characteristics of the proposed FCL system were investigated experimentally by use of a small test SCFCL of a transformer type (L-type) with a resistor and a $\mathrm{ZnO}$ device. The proposed FCL system switches alternatively L-type and L+R-type in a cycle. Relations among the threshold voltage of $\mathrm{ZnO}$ device (varistor voltage), the current limiting impedance and the resistance of the resistor were discussed.

\section{PROPOSED FCL SYSTEM}

\section{A. Inductive (L) Type FCL and Resistive (R) Type FCL}

Many types of SCFCLs are studied for the reliability of the electric power system. They can be classified into two types by their current limiting impedances. The SCFCLs which limit the fault currents by their inductances are called L-type, and which limit the fault currents by their resistances are called R-type. Both types of SCFCL have advantages and disadvantages.

L-type SCFCL has good features that the heat generation by the current is very small during its current limiting operation, and then it can recover to the waiting mode from the current limiting mode in a shorter time than R-type one. And, in general, since the impedance of a power system is inductive, L-type SCFCL can suppress the AC component of fault current better than R-type one of the same impedance as is shown in Fig. 1.

Disadvantages of L-type SCFCL are that it can not suppress the DC component of the fault current and the large over-voltage across the SCFCL appears at the start of the current limiting operation. 


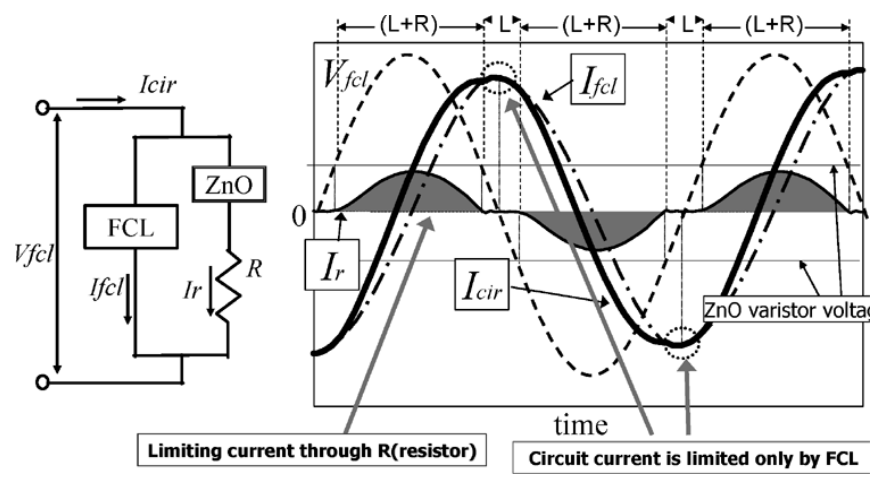

Fig. 2. Basic concept of the proposed FCL system, which switches L-type and $\mathrm{L}+\mathrm{R}$ type alternatively.

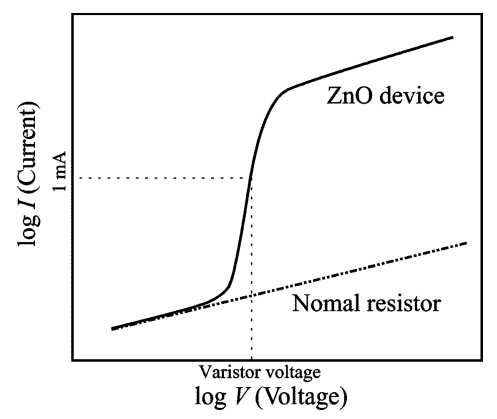

Fig. 3. Typical current-voltage characteristics of a $\mathrm{ZnO}$ device.

On the other hand, R-type SCFCL has advantages that it can suppress the DC component of the fault current better and has simpler and smaller structure than L-type one in general.

Disadvantages of R-type SCFCL are that it needs more time to recover to the waiting mode from the current limiting mode than L-type SCFCL, because of the heat generation at the fault and it might be burnt out by the heat generated by the fault current.

\section{B. Basic Concept of Proposed FCL System}

The schematic figure of the SCFCL in parallel with a resistor and a $\mathrm{ZnO}$ device is shown in Fig. 2. A typical current-voltage characteristic of the $\mathrm{ZnO}$ device is shown in Fig. 3. A ZnO device has high resistance when the voltage across it is lower than a certain value (off-state), and low resistance when the voltage is higher than a certain value (on-state). This boundary voltage is called "varistor voltage". Then a $\mathrm{ZnO}$ device is installed as a switching device.

When the SCFCL is in the waiting mode, because of the small impedance of the SCFCL, the voltage $V_{f c l}$ across the SCFCL is so small that the $\mathrm{ZnO}$ device is off-state and the current does not flow through the resistor. When a fault occurs, the SCFCL turns into current limiting mode and becomes of high impedance. The voltage across the SCFCL exceeds the threshold voltage (varistor voltage) of the $\mathrm{ZnO}$ device. It turns to be on-state and the fault current flows also through the $\mathrm{ZnO}$ device and the resistor. Consequently the fault current is limited not only by the

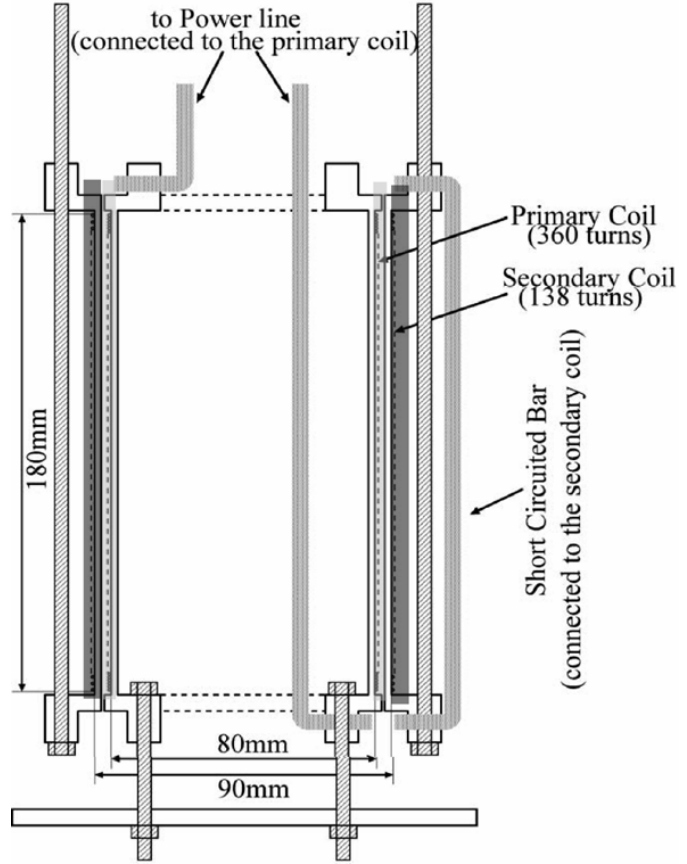

Fig. 4. One of the test 3-phase SCFCL of transformer type (primary coil inductance $3.82 \mathrm{mH}$, secondary coil inductance $0.69 \mathrm{mH}$, mutual inductance $1.43 \mathrm{mH})$.

SCFCL but also by the resistor. During one cycle of the voltage, the $\mathrm{ZnO}$ device is on-state around the peak of the voltage, and the current flows through both the SCFCL and the resistor. A certain amount of excessive energy is dissipated in the resistor. The $\mathrm{ZnO}$ device is off-state around the peak of the current which is suppressed only by the SCFCL (L-type). Finally the proposed FCL system switches L-type and L+R-type in a cycle alternatively.

\section{EXPERIMENTAL SYSTEM}

\section{A. Test SCFCL of Transformer Type}

The three-phase SCFCL unit contains three SCFCLs of transformer type in one cryostat [4]. Only one of the three SCFCL was used for the basic tests. As shown in Fig. 4, one of the SCFCL consists of two superconducting coils magnetically coupled co-axially. The inner (primary) coil will be connected to a power line. The outer (secondary) coil is superconductively short-circuited. When both superconducting coils are in the superconducting state, the magnetic flux is cancelled and the reactance of the SCFCL at the primary coil terminal is small (just a leakage reactance). When the fault current reaches the trigger current level, the super/normal transition occurs only in the secondary coil. The reactance of the SCFCL becomes large (almost equal to that of the primary coil $=1.2 \mathrm{Ohm}$ ) and the fault current is limited.

The superconducting wire of the primary coil is made of twisted three $\mathrm{CuNi} / \mathrm{Cu} / \mathrm{NbTi}$ (3.3:0.3:1) strands. The secondary coil wire is made of $\mathrm{CuNi} / \mathrm{NbTi}$ (4.3:1) strands. 


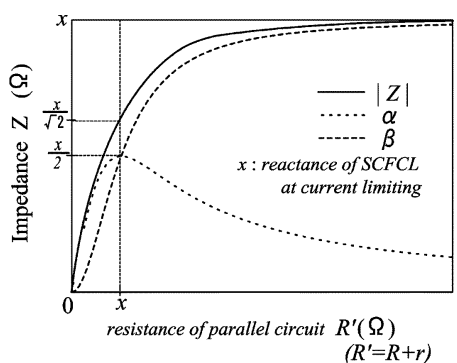

Fig. 5. Impedance of the proposed FCL system as a function of the resistance of the parallel resistor.

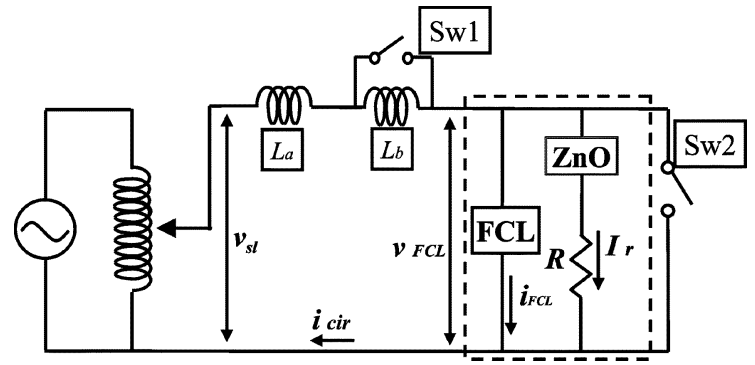

Fig. 6. Experimental circuit.

\section{B. Total Impedance of the FCL System and the Resistor}

The total impedance $Z$ of the SCFCL in parallel with a resistor and a $\mathrm{ZnO}$ device is defined as follows,

$$
Z=\alpha+j \beta \equiv|Z| e^{j \theta}=\left(\frac{1}{R+r}+\frac{1}{j x}\right)^{-1},
$$

where $R$ and $r$ is the resistance of the resistor and the $\mathrm{ZnO}$ device, respectively, $x$ is the reactance of the SCFCL. The impedance of the SCFCL is assumed to be completely inductive.

The total impedance of the FCL system in the current limiting mode is shown in Fig. 5 as a function of the resistance $R^{\prime}=$ $R+r$ of parallel circuit. The resistive component $\alpha$ has the maximum value at $R^{\prime}=x$ where the features of the R-type FCL appears most strongly. Therefore the resistor of 0.5, 1.0, 2.0 and 4.0 Ohm are selected for the tests of the model FCL system, because the impedance of the SCFCL at the current limiting mode is $1.2 \mathrm{Ohm}\left(=x_{\max }\right)$.

\section{Experimental Method}

In order to investigate the fundamental characteristics of the inductive SCFCL in parallel with a resistor and a $\mathrm{ZnO}$ device, the experiments using a single-phase test circuit (Fig. 6) were carried out.

The experimental circuit consists of two reactors $L_{a}$ and $L_{b}$, whose inductances are $2.13 \mathrm{mH}$ and $6.40 \mathrm{mH}$ respectively, a variable voltage transformer (slidac) and the SCFCL in parallel with a resistor and $\mathrm{ZnO}$ device are connected in series. A shortcircuit switch Sw1 is connected in parallel to the reactor $L_{b}$ to simulate a fault by closing it for $100 \mathrm{~ms}$. And switch Sw2 is closed to short-circuit the SCFCL for 0.9-2.0 s in order to make

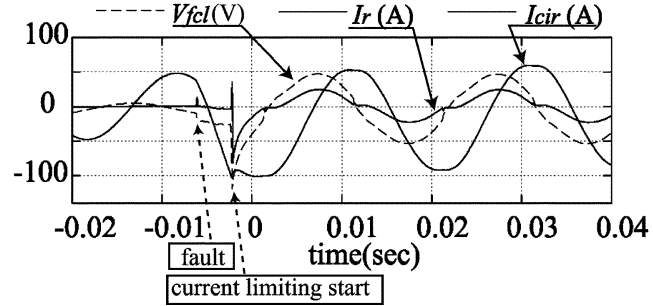

Fig. 7. Experimental results of the current limiting operation of the proposed FCL system. (Varistor voltage of $\mathrm{ZnO}$ device is $22 \mathrm{~V}$, the resistance of the resistor is $2 \mathrm{Ohm}$ ).

the SCFCL recover to the waiting mode. A commercial power supply was used as AC power source, and the rated voltage and frequency are $\mathrm{AC} 220 \mathrm{~V}$ and $50 \mathrm{~Hz}$. The output voltage of the slidac was coordinated at AC $100 \mathrm{~V}$.

In the SCFCL in parallel with a resistor and a $\mathrm{ZnO}$ device, ceramic ZnO devices (ENC220-54 [Fuji Electric Device Technology Co.]) for $22 \mathrm{~V}$ of varistor voltage is used for the $\mathrm{ZnO}$ device, and non flammable wire-wound fixed resistors (Japan Resistor Mfg. Co.) of $1 \mathrm{Ohm}$ and $2 \mathrm{Ohm}$ are used. The resistance $R$ was varied from 0 to $4 \mathrm{Ohm}$.

\section{EXPERIMENTAL RESULTS AND DISCUSSION}

\section{A. Experimental Results}

One of the experimental results is shown in Fig. 7. The simulated fault occurs at $-6 \mathrm{~ms}$. About $4 \mathrm{~ms}$ after that, the SCFCL starts to limit the fault current and the voltage $V_{f c l}$ across the FCL exceeds the varistor voltage $(22 \mathrm{~V})$, the $\mathrm{ZnO}$ device is on-state and the current $I_{r}$ flows through the $\mathrm{ZnO}$ device and the resistor. At the time around $2 \mathrm{~ms}$, the circuit current $I_{\text {cir }}$ has peak value. Because the voltage $\left|V_{f c l}\right|$ is less than the varistor voltage, the $\mathrm{ZnO}$ device is off state and the fault current is limited only by the reactance of the SCFCL. That is L-type current limiting mode. Soon after that, $V_{f c l}$ exceeds the varistor voltage and the resistor current starts to flow again. A certain amount of energy is consumed at the resistor. That is L+R type current limiting mode. These current limiting modes switch each other alternatively during the current limiting operation. The current $I_{r}$ flows intermittently.

\section{B. Resistor and Energy Consumption}

It is confirmed that the trigger current level is independent from the resistor, because the fault current does not flow through the resistor before the start of the current limiting operation of the SCFCL. The waveforms of the circuit current $I_{c i r}$ for each resistance $R$ and the first peak value of $I_{c i r}$ after the current limiting operation of SCFCL for $R$ are shown in Fig. 8. The peak value of the circuit current becomes constant for larger $R$ than about $2 \mathrm{Ohm}$, where the fault current is limited only by the reactance of the SCFCL around the peak of the current. However, the resistance $R$ is so small that the current $I_{r}$ becomes large and affects the peak value of $I_{c i r}$.

On the contrary, the power $P_{r} \mathrm{~kW}$ consumed and the energy $W_{r} \mathrm{~J}$ dissipated within one cycle at the resistor are larger for the smaller resistance $R$ as shown in Fig. 9. 

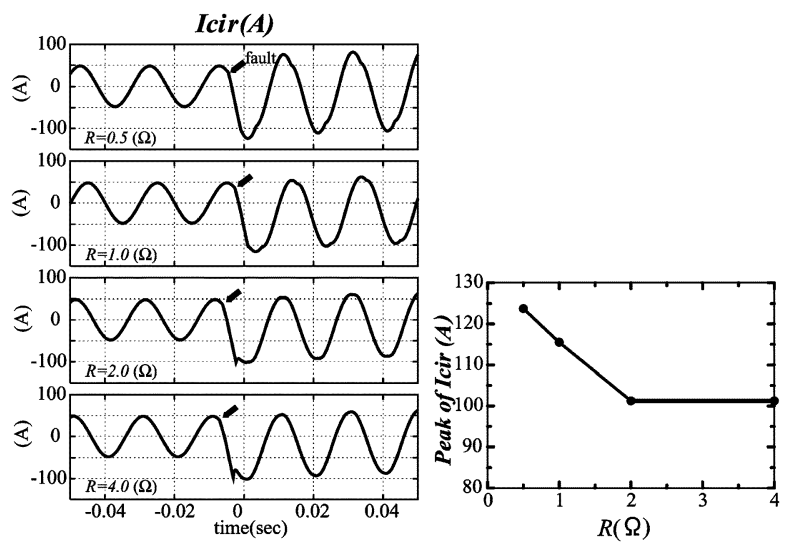

Fig. 8. Circuit current at the current limiting operation and its peak value for various resistance of the resistor.
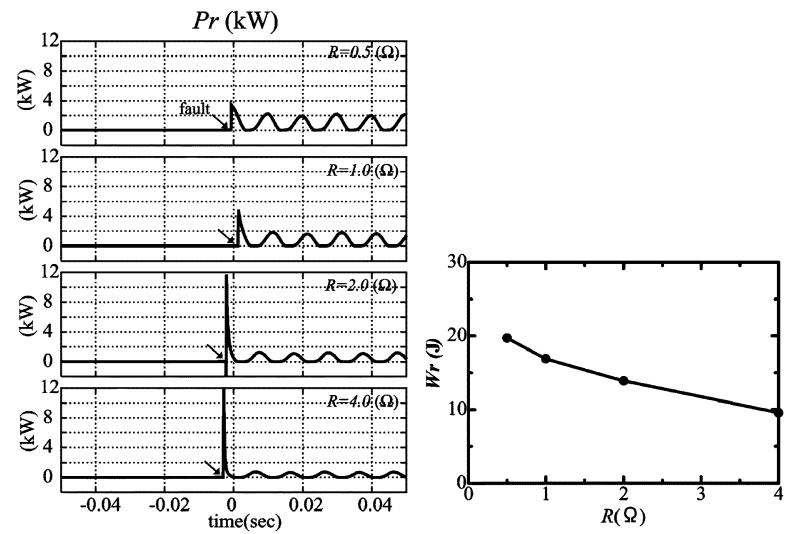

Fig. 9. Power consumed $P_{r}$ and energy dissipated $W_{r}$ at the resistor during the current limiting operation for various resistance of the resistor.

Therefore the adequate value of the resistance for good performance in both the current limiting and the energy absorption exists and is about $2 \mathrm{Ohm}$ in the test case.

\section{CONCLUSION}

A new FCL system which consists of the inductive SCFCL in parallel with a resistor and a $\mathrm{ZnO}$ device is proposed. A model system was made and the fundamental tests were carried out. Throughout the experiments, the following results were obtained.
1) The proposed FCL system has both L-type and R-type current limiting characteristics. The current limiting characteristics of L-type and R-type appear alternately in a cycle.

2) From the viewpoint of the fault current limiting function, the resistance of the parallel resistor must be larger than a certain value (about $2 \mathrm{Ohm}$ for the test) so that the current peak is limited only by the reactance of the SCFCL. On the contrary, larger energy absorption in the FCL system at the fault is expected for the power system stability. The total energy consumed at the resistor for one cycle at the current limiting operation is larger for the smaller resistance.

3) Therefore, the optimum value of the resistance for good performance in both the current limiting and the energy absorption exists and is about $2 \mathrm{Ohm}$ in the test case.

4) With appropriate set of these parameters, the proposed FCL system can suppress the peak of the fault current and also can dissipate the excessive energy of the power system.

5) It was confirmed the proposed FCL system represents desirable current limiting characteristics.

6) $\mathrm{A} \mathrm{ZnO} \mathrm{device} \mathrm{of} \mathrm{the} \mathrm{large} \mathrm{energy} \mathrm{capacity} \mathrm{should} \mathrm{be} \mathrm{de-}$ veloped for scaling up the proposed FCL system.

The proposed FCL system of 3-phase type will be tested in a power system simulator to investigate the features in improvement of the power system stability.

\section{REFERENCES}

[1] H. J. Boenig and D. A. Paice, "Fault Current Limiter using a superconducting coil," IEEE Trans. Magn., vol. 19, no. 3, pp. 1051-1053, May 1983.

[2] R. Kreutz et al., "System technology and test of CURL 10, a $10 \mathrm{kV}$, 10MVA resistive high-Tc superconducting fault current limiter," IEEE Trans. Appl. Supercond., vol. 15, no. 2, pp. 1961-1964, Jun. 2005.

[3] T. Yazawa et al., "66kV/1 kA high-Tc superconducting fault current limiter magnet," IEEE Trans. Appl. Supercond., vol. 15, no. 2, pp. 2059-2062, Jun. 2005.

[4] H. Hatta, T. Nitta, S. Muroya, Y. Shirai, and T. Kitagawa, "Experimental study on sudden-short-circuit characteristic of synchronous generator with SCFCL," IEEE Trans. Appl. Supercond., vol. 11, no. 1, pp. 2343-2346, Mar. 2001.

[5] Y. Shirai et al., "Study on recovery time of a superconducting fault current limiter with adjustable trigger current level," IEEE Trans. Appl. Supercond., vol. 11, no. 1, pp. 2086-2089, Mar. 2001.

[6] _ , "Recovery characteristics of fault current limiter with adjustable trigger current level," IEEE Trans. Appl. Supercond., vol. 9, no. 2, pp. 1381-1384, Jun. 1999.

[7] — , "A proposal of new operating procedure of transformer type fault current limiter," IEEE Trans. Appl. Supercond., vol. 12, no. 1, pp. 885-889, Mar. 2002.

[8] - "Over-voltage suppression in a fault current limiter by a $\mathrm{ZnO}$ varistor," IEEE Trans. Appl. Supercond., vol. 13, no. 1, pp. 880-883, Jun. 2003. 